\title{
LA PALABRA A LOS OYENTES: LOS COMENTARIOS DEL PÚBLICO EN UN CUESTIONARIO SOBRE LA PERCEPCIÓN DE LA CALIDAD DE LA INTERPRETACIÓN CONSECUTIVA DE ESTUDIANTES EN PRÁCTICAS ${ }^{1}$
}

\author{
Elena Errico \\ eerrico@uniss.it \\ Università di Sassari \\ Mara Morelli \\ mara.morelli@unige.it \\ Università di Genova
}

\section{Resumen}

Esta contribución se inscribe en un proyecto en el que estamos investigando la percepción de la calidad por parte de los usuarios de un servicio de interpretación consecutiva (IC) español-italiano desarrollado por estudiantes en prácticas. Desde el punto de vista teórico-metodológico, hemos adoptado la perspectiva etnográfica (Angelelli 2004; Valero-Garcés \& Martin 2008) y utilizado una combinación de herramientas investigadoras que incluye un cuestionario para cuyo diseño nos hemos guiado por el desarrollado por Collados Aís (1998). Hasta la fecha contamos con 403 cuestionarios recogidos en 14 actos diferentes. En esta ocasión nos centramos en la sección del cuestionario llamada "Más comentarios" que pueden rellenar los oyentes al final. Nos interesa ver con qué frecuencia se agregan comentarios, de qué tipo son y si resultan coherentes con el resto del cuestionario. Además, veremos si los que atribuyen juicios y notas negativas en el cuestionario añaden detalles en la sección "Más comentarios".

1. El artículo es el resultado del trabajo conjunto de las dos autoras. Sin embargo, la redacción de los primeros cuatro apartados corre a cargo de Elena Errico, mientras que los restantes fueron escritos por Mara Morelli. 


\begin{abstract}
"The listener's perspective: audience comments attached to a questionnaire on the perception of quality in consecutive interpreting assignments performed by trainees"

This study is part of a project investigating user-perceived quality of consecutive interpreting assignments carried out as part of an on-the-job training scheme offered to our students. From a theoretical-methodological point of view we have adopted the ethnographic perspective (Angelelli 2004; Valero-Garcés \& Martin 2008) and administered users and other participants a questionnaire replicated from Collados Aís's research (1998). Thus far, we have collected responses from 403 informants in 14 different events. In this paper we focus on the "Additional Comments and Suggestions" section which the informants can fill in at the end of the questionnaire. We examine the types of commentaries added, their frequency and consistency with the rest of responses. We also explore whether the informants who provide negative assessments in the questionnaire add details in this section.
\end{abstract}

Palabras clave: Comentarios del público. Interpretación consecutiva. Par de lenguas español-italiano. Estudiantes en prácticas. Percepción de la calidad.

Keywords: Audience comments. Consecutive interpreting. Spanish-Italian language pair. Trainees. Perception of quality.

Manuscript received on February 15, 2013 and accepted for publication on January $19,2014$. 


\section{Introducción: el contexto formativo}

El presente estudio pretende compartir los resultados de un proyecto en el que estamos investigando la percepción de la calidad por parte de los usuarios de un servicio de interpretación consecutiva (IC) desarrollado por estudiantes en prácticas. En este momento contamos con 403 cuestionarios (excluidos los de autoevaluación) recogidos en 14 actos diferentes. Después de haber presentado los resultados generales de nuestra investigación en su primera fase (Errico \& Morelli 2013) y un estudio de caso (Errico \& Morelli 2012), en esta ocasión profundizamos en una sección del cuestionario concreta, la de "Más comentarios", que pueden rellenar los oyentes al final. Nos interesa ver con qué frecuencia se agregan comentarios, de qué tipo son y si resultan coherentes con el resto del cuestionario.

Nuestro contexto laboral como profesoras es un Corso di Laurea Magistrale ${ }^{2}$ de la Universidad de Génova, donde impartimos técnicas de interpretación, sobre todo IC, en el par de lenguas español-italiano por un total de 100 horas lectivas. A lo largo de la carrera, se le ofrece al alumnado la oportunidad de hacer prácticas de interpretación en situaciones auténticas, sobre todo seminarios y cursos de formación en mediación comunitaria en los cuales intervienen expertos hispanohablantes. Estas prácticas se han convertido en una parte fundamental de nuestro proyecto pedagógico, inspirado en el planteamiento sociocontructivista aplicado a la pedagogía de la interpretación (Morelli 2008; 2010). En consonancia con este enfoque, centrado en la participación activa y consciente del discente en el proceso de aprendizaje, que se concreta también brindándole oportunidades "[of] authentic situated action, collaborative construction of knowledge, and personal experience" (Kiraly 2000: 3), consideramos sumamente útil exponer a los estudiantes a situaciones auténticas para que puedan poner a prueba habilidades que deberán funcionar en un marco profesional más allá del aula.

2. En el sistema universitario italiano, los Corsi di Laurea Magistrale son estudios de segundo ciclo a los cuales se accede tras completar un primer ciclo de tres años de duración (Corsi di Laurea Triennale, 180 ECTS). La titulación se obtiene realizando 120 ECTS en dos cursos. 
Puesto que uno de los planteamientos fundamentales de la pedagogía socioconstructivista es fomentar la autonomía del discente, procuramos en todo momento animar a los alumnos a que se responsabilicen de sus elecciones. En el caso concreto de las prácticas, no son las profesoras las que escogen a los aspirantes a intérpretes, sino que cada vez que se presenta una oportunidad de práctica, se lanza una convocatoria para que se ofrezcan voluntarios. Hasta el momento el sistema ha funcionado, ya que nunca se han presentado intérpretes totalmente incompetentes. La valoración del público ha sido positiva la mayoría de las veces $\mathrm{y}$, aun cuando ha habido retroalimentación negativa por parte de los oyentes, los estudiantes implicados no se han sentido hundidos por la experiencia, sino que en ciertos casos la han aprovechado como oportunidad para mejorar, hasta el punto de aceptar participar en prácticas posteriores (Errico \& Morelli 2012). De esta manera, aunque no todas las interpretaciones han sido exitosas en las valoraciones del público, podemos afirmar que sí lo han sido en el marco de nuestro proyecto pedagógico.

El material que estamos recopilando cumple fundamentalmente dos propósitos: en primer lugar, es útil para probar la eficacia de nuestras estrategias docentes y la reacción de nuestros estudiantes cuando interpretan ante un público. En segundo lugar, la posibilidad de disponer de material auténtico, aunque sea de sujetos en formación, nos ha permitido ir creando un archivo de documentación para estudiar la reacción del público ante los intérpretes, es decir, su percepción de la calidad del producto en situaciones concretas.

\section{Los antecedentes de la investigación de la calidad en interpretación}

La investigación sobre calidad en interpretación en sus albores privilegió el estudio de las expectativas, es decir, cómo debería ser una interpretación ideal prescindiendo de las variables situacionales (cf., por ejemplo, Bühler 1986; Kurz 1993; Moser 1995; Chiaro \& Nocella 2004). ${ }^{3}$ Estos estudios, a pesar de presentar un panorama muy heterogéneo según el tipo de encuestados, coinciden fundamentalmente en identificar el contenido como criterio principal de valoración, aunque no dicen nada sobre cuál sería la reacción efectiva de los destinatarios ante una interpretación concreta.

Los primeros trabajos sobre la evaluación de interpretaciones concretas se los debemos a Gile (1990; 1995), que plantea la posibilidad de un desfase entre expectativas y evaluación, además de cuestionar la fiabilidad de los

3. Para un repaso exhaustivo de la literatura sobre la percepción de la calidad en interpretación desde una perspectiva multidisciplinar, véase Collados Aís et al. (2011). 
usuarios como evaluadores. Se inscribe en esta línea la investigación pionera de Collados Aís (1998), la cual demuestra empíricamente que la conducta de los usuarios contradice lo que declaran respecto a la primacía del contenido, pues en su experimento sobre la evaluación de una interpretación simultánea, la monotonía de la voz incide negativamente en la valoración global de la interpretación, mientras que una voz melodiosa propicia una valoración positiva a pesar de la transmisión incorrecta del sentido (que no es detectada). Garzone (2003) identifica, en un estudio piloto, la interferencia negativa de una prosodia deficiente en la evaluación, pese a las expectativas declaradas de los mismos informantes, que sitúan la forma en las últimas posiciones.

En años recientes la percepción de la calidad en interpretación se ha investigado de forma más sistemática y rigurosa. Collados Aís et al. (2007) amplían y afinan un planteamiento experimental complejo y riguroso ya adoptado en Collados Aís (1998), aplicándolo a 11 parámetros. ${ }^{4}$ Los resultados experimentales corroboran los de estudios anteriores, identificando una mayor incidencia de los rasgos no verbales en la evaluación de la calidad, aunque son justamente estos parámetros los que más variabilidad manifiestan (Collados Aís et al. 2007: 218-219). Por otra parte, el orden de prioridades se mantiene a la inversa para las expectativas, en las que los informantes siguen privilegiando los parámetros verbales y de contenido, tal como la transmisión correcta y completa del sentido (Collados Aís et al. 2007: 220), a pesar de que se trata de parámetros que no se pueden controlar sin hacer una comparación con el texto fuente, lo cual es evidentemente imposible en interpretación simultánea por el solapamiento entre orador e intérprete, pero también problemático en IC (Gile 1995).

\section{Materiales y métodos: la investigación etnográfica}

El estudio que proponemos se basa, entre otros, en la distribución de un cuestionario. Para su diseño nos guiamos por el desarrollado por Collados Aís (1998). Puesto que en nuestro caso la modalidad empleada fue eminentemente la IC, llevamos a cabo algunos ajustes y añadidos teniendo en cuenta el carácter presencial de la misma para incorporar en el análisis el contexto situacional y las competencias de oratoria en público.

Además de la diferente modalidad de interpretación estudiada, la diferencia metodológica fundamental respecto a la investigación que hemos tomado

4. Los parámetros estudiados son: acento, agradabilidad de la voz, fluidez, cohesión, transmisión correcta del sentido, transmisión completa del discurso original, dicción, entonación, estilo, gramaticalidad y terminología. 
como referencia es que, como hemos mencionado, nuestros datos proceden de un contexto naturalista y no experimental y los analizamos a través de una combinación de herramientas. Los cuestionarios de respuesta cerrada, que los propios estudiantes también rellenan como autoevaluación, y las grabaciones, algunas en audio y otras en vídeo, nos permiten contar con un respaldo cuantitativo a nuestro análisis e identificar tendencias generales. A ellos hemos agregado herramientas etnográficas, ${ }^{5}$ tales como nuestra observación participante y comentarios libres tanto del público como de los propios aprendices, a quienes pedimos que comenten también la retroalimentación de la audiencia acerca de su prestación. En algunos casos los comentarios de los estudiantes se asemejan a auténticas narraciones personales de su experiencia. Además, utilizamos datos contextuales longitudinales sobre los aprendices, como su expediente académico en interpretación y nuestras notas de campo tomadas durante las clases.

Nos hemos orientado hacia la perspectiva etnográfica porque, al ser un conjunto de técnicas de recogida de datos abierto y flexible, nos puede ayudar a estudiar las especificidades de cada interpretación y de cada aprendiz en su contexto, investigando el papel que los estudiantes-intérpretes desempeñan, su reacción y las relaciones comunicativas que se establecen entre los participantes; en otras palabras, las relaciones discursivas que se negocian en un contexto dado. Este enfoque se compagina muy bien con la perspectiva pedagógica sociocontructivista, que hace hincapié en los factores individuales y no pretende necesariamente deslindar causas y consecuencias del aprendizaje, sino considerarlas como procesos que interactúan entre sí, de forma holística. Es más, la flexibilidad de la investigación de tipo cualitativo nos permite ir revisando sobre la marcha nuestros planteamientos, conforme se amplía el corpus y aportamos nuevos datos. Los datos de los que disponemos hasta la fecha nos permiten llevar a cabo una observación tanto horizontal, contrastando los distintos eventos, como longitudinal, aislando estudios de caso e integrándolos con información procedente de fuentes que no se ciñen al acto en sí, sino que dan cuenta del antes y, en ciertos casos, del después de la trayectoria formativa de nuestros estudiantes. Este conjunto de herramientas investigadoras y el tamaño del corpus que estamos estudiando constituyen, en nuestra opinión, condiciones propicias para recuperar y reclamar la importancia de reconstruir e interpretar la perspectiva subjetiva de los participantes

5. El planteamiento etnográfico ha sido aplicado a los Estudios de Interpretación en los trabajos de Angelelli (2004) y Valero-Garcés \& Martin (2008). 
en el estudio, ${ }^{6}$ sin sacrificar por ello los imprescindibles requisitos de rigor científico y transferibilidad de la investigación.

\section{Las situaciones comunicativas}

Tras haber enmarcado el contexto formativo y el planteamiento teórico-metodológico, cabe hacer un repaso de las situaciones comunicativas en las que interpretaron nuestros estudiantes.

El primer evento se remonta a mayo de 2010. En esa ocasión organizamos un congreso en Génova sobre la mediación (véase www.iberistica.unige. it, Archivio Comunicazioni, para los detalles del congreso). El congreso fue precedido por cinco talleres en los que se desarrollaron los siguientes temas: mediación comunitaria y seguridad pública, mediación comunitaria y redes vecinales, mediación intercultural, mediación en el ámbito sanitario y técnicas de mediación. ${ }^{7}$ Los destinatarios fueron unas 18 personas en cada taller procedentes de diferentes realidades: funcionarios públicos, operadores y asistentes sociales, psicólogos, médicos, educadores, lingüistas, etc. Todos los talleres fueron interpretados por una pareja de estudiantes de nuestra carrera.

El segundo evento seleccionado se celebró en Génova a mediados de octubre de 2010 y fue el Congreso Mundial de las Comunidades Terapéuticas, en el que participaron más de 200 personas procedentes de todo el mundo. En las sesiones plenarias del congreso estaba prevista la simultánea (por parte de AIIC), mientras que en los grupos de trabajo, integrados por unas 20 personas cada grupo, garantizamos la interpretación gracias a la disponibilidad de nuestra facultad y de nuestros estudiantes. En estos grupos de trabajo no había un auténtico ponente, sino diferentes moderadores. La situación era muy polifónica y polifacética en cuanto a la procedencia geográfica de los participantes y a su edad.

El tercer y el cuarto encuentro seleccionados son sesiones de formación de una jornada de duración cada uno, enmarcadas en un curso anual (20102011) organizado por la Fondazione e Associazione San Marcellino de Génova y por la Fondazione Culturale San Fedele, en colaboración con la Facultad de Ciencias Políticas de la Universidad de Bolonia. Los participantes eran unas 30 personas, la mayoría estudiantes universitarias. Dos estudiantes interpretaron

6. En palabras del antropólogo Duranti (2000: 86), la etnografía no es sino "un dialogo fra diversi punti di vista, compresi quelli delle popolazioni studiate, dell'etnografo e delle sue scelte teoriche".

7. A continuación los nombres de los expertos que condujeron los diferentes talleres: Ramón Alzate Sáez de Heredia, Carlos Giménez Romero, Alejandro Nató, Dora Sales Salvador y Juan Carlos Vezzulla. 
en todas las ocasiones del español al italiano y viceversa. Participaron con esta función un total de 7 estudiantes, ya que una repitió la experiencia en dos ocasiones diferentes. La primera fecha seleccionada en el curso fue el 23 de octubre de 2010, en la que el formador fue el sociólogo español Pedro Cabrera, de la Universidad Pontificia Comillas. El ponente es un buen orador, tiene una manera muy ordenada y pausada de presentar, la modalidad usada fue la IC tradicional. La segunda fecha seleccionada fue el 15 de enero de 2011, cuando intervino Pedro Meca, de la Asociación Compagnons de la nuit de París.

Los mismos dos formadores fueron interpretados también en el curso homólogo de 2011-2012. Los participantes en este curso fueron más de 50, siempre con una mayoría de estudiantes. A lo largo de ese mismo curso 20112012, participaron también dos operadores representantes de dos asociaciones españolas que trabajan con personas sin hogar y los datos referentes a esta jornada (febrero de 2012) constituyen parte de nuestro corpus.

Otro evento seleccionado fue una formación en mediación comunitariatransformación de conflictos llevada a cabo el 14 y el 15 de marzo 2011 en Génova. Los destinatarios fueron unas 25 personas representantes de un barrio conflictivo de la ciudad, pero que está insertado en un proyecto de recuperación urbana y social. La formación se celebró en una sala de la propia Casa de Barrio y participaron diferentes habitantes del barrio, un policía urbano, un basurero, operadores y trabajadores sociales, etc. Estaban representadas diferentes nacionalidades: italiana, tunecina, senegalesa, etc. El formador era Alejandro Nató, argentino, experto en mediación. La pareja de intérpretes, por primera vez, estaba formada por una estudiante 'avanzada' de segundo año de la Laurea Magistrale y por un estudiante 'novato' de primer año de la Laurea Magistrale. La técnica que se utilizó con mayor frecuencia fue la IC, con o sin toma de notas.

Tres eventos (cursos de formación en mediación) se destinaron a los funcionarios de la Policía Municipal en marzo de 2011, mayo de 2011 y febrero de 2012, respectivamente. Salvo en el curso de mayo, donde los formadores fueron Javier Vidargas, mediador mexicano, y Alejandro Nató, el instructor de esta parte del proyecto fue Juan Carlos Vezzulla, argentino. Los participantes oscilaron entre los 15 y los 25 , según el mes del curso.

En noviembre de 2011 se organizó la tercera fase del proyecto genovés de mediación y se capacitó en mediación educativa a un grupo de 25 personas. El curso se llevó a cabo en una escuela de la periferia genovesa. Los participantes procedían tanto del mundo escolar como del deportivo y del social. El instructor fue Ramón Alzate, experto en mediación, vasco. 
Los últimos actos que conforman nuestro corpus son las tres sesiones, de tres jornadas cada una, de la edición de 2012 del curso de concienciación para la mediación comunitaria, en el cual intervino Alejandro Nató como instructor, destinadas a unos 70 operadores (trabajadores sociales, asistentes sociales, mediadores interculturales, policías locales, educadores, psicólogos, vecinos, etc.), muchos de los cuales habían participado en algunas jornadas de capacitación anteriores que constituyen parte de nuestro corpus.

Puesto que el público era diferente y más numeroso, no hemos entregado nunca el cuestionario durante los congresos que hemos ido organizando todos los años en mayo, desde 2009, siempre con la presencia de estudiantesintérpretes en prácticas. Además, cambia la situación comunicativa y, sobre todo, la intervención de los intérpretes es mucho más puntual (solo para las IC de los invitados hispanohablantes). Agregaremos más comentarios sobre la posibilidad de utilizar también estos contextos en la parte final de este trabajo.

\section{Análisis general de los comentarios en los márgenes de los cuestionarios}

En primer lugar haremos referencia a los comentarios generales que recogimos en los talleres de mayo de 2010 (véase apartado anterior) puesto que nos ayudaron para elaborar el cuestionario en el que basamos nuestros análisis siguientes. En el taller de mediación intercultural, con unos 18 participantes, muchos de ellos mediadores interculturales e investigadores bilingües español-italiano, encontramos los siguientes comentarios, aquí traducidos del italiano:

Poner un intérprete capaz de captar las diferencias léxicas básicas para reducir los tiempos de traducción sucesiva y selectiva que no penalice a ninguno de los participantes.

Fue muy interesante pero no había intérprete y, por tanto, se entendía con dificultad al profesor.

Este segundo comentario es sumamente interesante porque es probable que sea debido a una disposición inadecuada de los intérpretes en el aula. Recordamos que, como la mayoría de los asistentes entendían bien ambas lenguas, los dos estudiantes intérpretes se habían sentado un poco alejados. Cuando llegó una mediadora intercultural de área africana, se sentó lejos de ellos y es probable que no les reconociera como intérpretes.

En el taller de mediación en el ámbito sanitario, uno de los asistentes nos dejó el siguiente comentario: 
Algunos problemas con la lengua y, por consiguiente, algún bajón en la concentración.

Este hecho (tener que escuchar en otra lengua y, luego, la interpretación con consiguiente pérdida de concentración) es un aspecto que puede ser muy interesante y en él que profundizaremos más adelante.

Si pasamos al análisis del corpus de los cuestionarios sobre la calidad percibida de los estudiantes-intérpretes en prácticas, reiteramos que en noviembre de 2012 (fecha en la que empezamos la redacción del presente trabajo) contábamos con 403 cuestionarios rellenados (excluyendo los de autoevaluación de los estudiantes, que merecerían un estudio aparte $)^{8}$ que incluyen 62 comentarios totales, lo cual corresponde a un 15,3\% del total. En algunos eventos no tenemos ningún comentario, mientras que el porcentaje máximo de comentarios en un evento sencillo es del $25 \%$.

Incluso cuando los destinatarios y el formador son los mismos (por ejemplo, en el caso del curso de concienciación para la mediación comunitaria en el que el cuestionario se entregó tres veces, al finalizar las tres jornadas de cada sesión), no consta ninguna regularidad entre el porcentaje de comentarios en una sesión y otra (oscila entre el $17,8 \%$ y el $8 \%$ ). Sin embargo, un aspecto que no podemos descartar y que queda como variable que puede sesgar los datos recogidos es la prisa o la escasa atención con la que los asistentes podrían haber rellenado los cuestionarios antes de marcharse.

Si pasamos al análisis general de las características señaladas como positivas en los comentarios, podemos identificar las siguientes: un servicio puntual, apropiado y adecuado; el hecho de que se perciba que los estudiantes aman este trabajo; la importancia y necesidad de que empiecen a ponerse a prueba en el mundo del trabajo; sus ganas de mejorar; el interés, la empatía, la profesionalidad y la fiabilidad que transmiten al interpretar. Por el contrario, estos son los aspectos destacados con más frecuencia en los comentarios negativos: en primer lugar, los rasgos paraverbales relacionados con la voz y, más concretamente, el volumen bajo, la velocidad excesiva y la mala vocalización.

$\mathrm{Si}$, en cambio, nos centramos en algunos comentarios concretos, el primer aspecto que llama la atención al analizar los comentarios, y también el más frecuente, es la diferencia de 'nivel' y de 'calidad' entre los dos intérpretes, algo que está muy recalcado en determinados eventos, uno de los cuales ya nos sirvió para presentar un estudio de caso en otra ocasión (Errico \& Morelli

8. Justamente cuando estamos a punto de entregar este trabajo para la publicación, se acaba de entregar para su próxima defensa el proyecto de fin de grado de una estudiante nuestra que empieza esta línea de investigación que creemos puede ser muy prometedora. 
2012). Se trata de nada menos que de 29 comentarios de este tipo y, como promedio, constituyen, en determinados eventos, más del $80 \%$ del total de comentarios agregados. A continuación presentaremos algunos ejemplos: ${ }^{9}$

La calidad percibida es muy diferente: una que deja el tiempo para elaborar el discurso; la otra que traduce muy rápida, con consiguiente pérdida, a veces, de claridad y comiéndose las palabras durante la traducción

o también:

La calidad de la interpretación no es homogénea. Uno de los dos intérpretes era muy claro y profesional; mientras que la otra era más insegura, menos fiel y con menor adecuación en lo estilístico.

Numerosos oyentes, también en el caso en que estuviera presente una de las dos investigadoras, lamentaron este aspecto, diciendo que sería mejor dividir el cuestionario en dos, previendo la evaluación de cada uno de los intérpretes por separado. En estas situaciones, la investigadora tuvo la oportunidad de explicar la importancia del tándem de trabajo en interpretación, de la dificultad, pero trascendencia, de la evaluación global del servicio por parte del público y de la posibilidad de agregar comentarios en los márgenes del cuestionario en la sección que estamos analizando.

Aparte de los comentarios contrastivos sobre la calidad de cada intérprete, el desglose general atestigua cierta igualdad entre comentarios apreciativos y negativos. Una vez más, también en esta ocasión, encontramos algunos comentarios mixtos, según se dirijan a una intérprete u otra. A continuación se facilita un ejemplo:

La intérprete de Toscana me inspiró más confianza y profesionalidad, aunque su acento muy marcado y su pronunciación me distraían mucho. La otra intérprete me gustó más por el entusiasmo y la idea de implicación que transmitía. Lástima que omita e 'interprete’ demasiado. La veo más adecuada para otros tipos de encuentros.

Interesante notar cómo, en dos de los comentarios anteriores, se ponen de relieve dos aspectos relacionados con lo paraverbal: la velocidad de elocución y la variante diatópica acentual. Además, en el otro comentario arriba indicado se hace referencia al concepto de fidelidad con respecto al original, aunque no sabemos si el destinatario tenía acceso directo y conocimiento profundo de la lengua origen.

Nos interesaba también observar si había comentarios estrechamente vinculados con la técnica, pero no son frecuentes. Entre los positivos, encontramos

9. Todos los ejemplos de comentarios están traducidos del italiano al español por las autoras. 
los siguientes: el sentido común de omitir cuando se entiende que el concepto está claro y la toma de notas con capacidad de síntesis; mientras que entre los negativos destacan el uso inadecuado (alejado) del micrófono y las pausas excesivas para pasarse el turno entre intérpretes. No obstante, este comentario se encuentra repetido, pero solo en una ocasión concreta. A continuación ponemos un par de ejemplos de cómo se verbalizan dichos comentarios, tanto positivos como negativos:

Enhorabuena: muy buenas. Creo que tuvieron el sentido común de no traducir cuando entendían que el formador había entendido. De no haberlo hecho, resultaría muy pesado. Repito mi buena impresión global: ¡Gracias! Lo que me gustó fue que consiguieran traducir amplios fragmentos sirviéndose de notas escritas lo que le permitió al ponente no interrumpirse demasiado.

Y finalmente:

Resultaban pesadas las pausas entre la toma de palabra de una intérprete cuando la otra le cedía el micrófono. Se interrumpía el ritmo.

También son escasos los comentarios acerca del contenido puntual (menos del $10 \%$ del total) y todos negativos. Pasando en reseña los mismos y yendo de lo general a lo particular, podemos subrayar algunos aspectos relacionados con la escasa familiaridad de los intérpretes con los temas tratados, con cierto fastidio al percibir alguna pérdida de partes del discurso o términos incoherentes en italiano, además de la sugerencia de intentar no traducir palabras, sino el significado del discurso ("Vistos los temas tratados, necesario un mayor conocimiento de la materia sin limitarse a traducir las palabras; entender el significado del discurso").

Por lo que se refiere a palabras o términos concretos, los comentarios nos dejan algunas sugerencias relativas a términos con significado parecido, pero valor diferente en las dos lenguas ("Prestar atención a unos términos que, aunque tienen un significado parecido en las dos lenguas, tienen un valor diferente, por ejemplo: cliente, mutua"), un malentendido acerca del verbo 'contener' (en la acepción utilizada en lo social) y una excesiva repetición del uso incorrecto de la muletilla appunto (justamente).

Otra variable que puede resultar interesante es analizar los comentarios según la profesión de quienes comentan (en caso de constar) para entender si la misma es significativa. Notamos que hay eventos donde, por ejemplo, los funcionarios de la Policía Local no agregaron ningún comentario. Sin embargo, cabe poner de relieve que los mismos funcionarios sí agregaron comentarios en los eventos no exclusivamente destinados a ellos (el curso de concienciación para la mediación comunitaria). También puede haberse debido al hecho de que las mismas personas ya tenían experiencia con el 
servicio de interpretación por ser el segundo año que participaban en una formación en mediación.

Por lo general, las notas y los comentarios más positivos proceden de psicopedagogos, sacerdotes, educadores, investigadores, profesores, asistentes sociales y mediadores interculturales (no siempre del área lingüística-cultural castellana). Más críticos, y también con notas negativas, parecen ser algunos operadores y asistentes sociales y algunas personas que se definen como profesionales libres. A veces, en presencia de notas o comentarios negativos, no se especifica ni la procedencia ni la profesión. Los estudiantes de otras carreras, sobre todo de trabajo social, oscilan entre la empatía, con notas muy positivas, y algunos comentarios duros y negativos. Por el momento, no podemos encontrar datos significativos en este tipo de desglose de comentarios por profesión declarada.

Por lo general, los comentarios parecen ser coherentes con las notas atribuidas a lo largo del cuestionario.

\section{Reflexiones sobre algunos elementos concretos}

El aspecto de la pérdida de concentración por problemas de lengua y por la interpretación, indicado en uno de los cuestionarios (véase apartado anterior), es un asunto en el que nos interesa profundizar. Somos conscientes de que, en la mayoría de los casos, muchas personas nativas italianas pueden intuir algo de lo que se dice en español y que puede ser pesado tener que volver a escuchar lo mismo en italiano. Cuando estamos presentes al empezar la sesión y presentamos a los intérpretes en prácticas, le comunicamos al público el tipo de técnica que usaremos, reconociendo que alarga los tiempos y que puede resultar aburrida. Sin embargo, a la vez, recordamos que en su trabajo podrían beneficiarse mucho de la doble escucha y de muchas de las técnicas que aplicamos los intérpretes. No olvidemos que casi todos trabajan o estudian en lo social y tienen un trabajo de índole relacional, policías incluidos. Volveremos sobre la importancia que para nosotras reviste este tipo de sensibilización en el apartado siguiente.

En nuestro estudio podemos contar con numerosos elementos que no pertenecen directamente al corpus presentado pero que no podemos descartar si queremos que el estudio no sea solo un producto sino un proceso y forme parte de un recorrido que tiene dos objetivos básicos: el de mejorar la calidad de la didáctica de la interpretación y el de sensibilizar constantemente a los destinatarios de un servicio de interpretación sobre la importancia de esta profesión, llevando a cabo una necesaria sensibilización sobre la complejidad de las tareas de interpretación para nuestros clientes. 
Efectivamente, a continuación presentamos algunos comentarios que los mismos oyentes nos hicieron después de su primera experiencia con la interpretación.

En un caso concreto, un policía participó como observador en otra edición de un curso de mediación en el que había participado hace dos años (tanto el formato como la duración no habían cambiado). Sin embargo, mientras tanto, este policía había asistido también al curso de sensibilización a la mediación comunitaria y, entonces, ya había escuchado la IC por parte de estudiantes en prácticas. En su informe de observador del curso analiza la lengua y a los intérpretes. A continuación facilitamos un ejemplo de sus comentarios:

Después de un primer, previsible, periodo de perplejidad durante el que nos interrogamos sobre cómo y para qué puedan servir las temáticas tratadas, mostrando una actitud crítica y casi de cierre, tal vez exacerbadas por la lengua del docente y por tener que recurrir a los traductores, la actitud va cambiando. [...] Superado el momento inicial de novedad y perplejidad ante la lengua extranjera, esta se convierte, en cambio, en un estímulo [...].

Otro ejemplo es el de las reflexiones que nos llegaron del curso dirigido a operadores para personas sin hogar que nos sirvió para el estudio del caso al que ya hemos hecho referencia en este trabajo (Errico \& Morelli 2012).

Una de las autoras de este trabajo intervino como formadora en la jornada final del curso de 2012. ${ }^{10}$ Durante la clase trajo a colación la mediación, presentando la interpretación interlingüística como forma de mediación y reiterando la importancia de los aspectos relacionados con el lenguaje y con las técnicas mediadoras en cualquier trabajo de tipo relacional. Más concretamente, la formadora remitió a los participantes al servicio de interpretación que habían recibido en anteriores clases. En la pausa, varias personas se le acercaron para darle más retroalimentación sobre lo que había pasado en una jornada concreta, recuperando muy positivamente el trabajo de los intérpretes, alabando la posibilidad que se les estaba dando a los estudiantes, entendiendo mejor qué supone la tarea de interpretar, también a la luz de las sucesivas experiencias de interpretación que habían tenido a lo largo del año y de lo que acabábamos de comentar sobre los procesos de interpretación del lenguaje.

Por último, presentamos el caso de una educadora y de dos asistentes sociales que habían tenido anteriormente una experiencia como usuarias del servicio de IC con estudiantes en prácticas. A la hora de organizar un taller

10. El tema tratado era "La mediación comunitaria y los territorios", clase impartida en copresencia con Danilo De Luise, de la propia Fundación organizadora del curso (véase www.iberistica.unige.it para más información sobre el proyecto) 
con los vecinos de un barrio sobre "Cómo mejorar mi barrio y la convivencia en él", estructurado según el esquema ya presentado (formador-facilitador hispanohablante interpretado por estudiantes en prácticas), la educadora y las asistentes sociales pensaron, en un principio, que se podría prescindir de la interpretación para evitar demasiadas superposiciones de voces y optimizar el escaso tiempo a su disposición, puesto que mucha de la interacción podría producirse entre los propios vecinos del barrio y que el formador tiene un conocimiento pasivo del italiano muy elevado. Sin embargo, al final de la formación dijeron que había sido muy importante que el intérprete estuviera presente y que fuera uno solo (la duración de los talleres lo permitía).

\section{Posibilidades de desarrollo futuro}

Creemos que son muchas las potencialidades de continuación y profundización de este trabajo, que aquí presentamos solo en términos de resultados generales, para ampliar el abanico de posibles horizontes por desarrollar.

Consideramos interesante, en esta segunda fase de nuestra investigación, introducir el concepto de las expectativas y experiencias previas del público con la interpretación y más concretamente con la técnica de la IC, lo cual puede ayudar a leer los datos de nuestro corpus con más detalle y hacia una mayor sistematización.

Otra posibilidad de estudio futuro que podría resultar fructífera puede proceder de la comparación entre los comentarios de los intérpretes en prácticas y los del público en los mismos eventos con el fin de observar discrepancias y convergencias y, en el caso de contar con las grabaciones en vídeo, volver a analizar fragmentos del evento más pormenorizadamente y centrándose en variables o parámetros específicos.

Además, sería interesante analizar en detalle si la autoevaluación del conocimiento del español por parte de los participantes tiene algún peso y de qué manera repercute, si es que lo hace, en las valoraciones.

Estamos pensando también en la oportunidad de diseñar y pilotar un cuestionario parecido al que se adjunta en el anexo y distribuirlo en los congresos que organizamos y que recurren a la IC, siempre por parte de estudiantes en prácticas, solo en intervenciones magistrales de máximo de una hora y media.

Por último, pero no menos importante, tenemos el objetivo de intentar organizar un taller de técnicas de interpretación destinado a los perfiles profesionales implicados en este estudio. Ya hemos anticipado en el apartado anterior el porqué de esta idea. Además, esta experiencia podría convertirse en un grupo de discusión para ahondar en expectativas y percepciones de los oyentes. 


\section{Conclusiones}

Nuestra experiencia nos ha permitido también observar algunas lagunas sustanciales tanto en el recorrido de muchos de los cursos en los que hemos trabajado como en la temática de la educación al cliente. En el caso concreto de la calidad de la interpretación, por ejemplo, consideramos que en la formación es más importante acompañar a los estudiantes hacia una autoevaluación continua, orientada según macroparámetros (parecidos a los que presentamos en el cuestionario, aunque menos desglosados), que asignar nosotros notas y juicios. Brindarles la posibilidad de ponerse a prueba en una palestra real y en un contexto profesional auténtico, aunque protegido, y de exponerse al juicio y a las valoraciones ajenas, es fundamental para nosotras. Y esto, necesariamente, significa exponerse a los que no saben, o sea, a los que a menudo saben del tema que se está tratando, pero que no saben de interpretación y de lenguas. Presentar sus comentarios y compartirlos en un trabajo como éste es una manera, para nosotras, de demostrar varias cosas. En primer lugar, la importancia fundamental de poder mezclar diferentes herramientas y metodologías a la hora de investigar y de poder recuperar los datos según queramos analizarlos. En segundo lugar, convertir en auténticos protagonistas del servicio prestado a los estudiantes y hacer partícipes a los destinatarios del servicio de interpretación de un enfoque de toma de responsabilidad mutua. En tercer lugar, llevar a cabo un trabajo que nunca toma la forma de un producto, sino que forma parte de un proceso constante en el que intentamos, según la situación y los objetivos, implicar a todos los componentes clave, sean ellos de índole docente, profesional o investigadora, que, en nuestro enfoque de investigación-acción participativa, no son aislables.

Finalmente, asumir que en los datos, afortunadamente, algo se nos escapará siempre del afán por ordenar, categorizar y sistematizar en el mundo, bastante imprevisible, del servicio de la interpretación, sometido a múltiples subjetividades y variables.

\section{Referencias bibliográficas}

ANGELELLI, Claudia. (2004) Revisiting the interpreter's role. A study of conference, court, and medical interpreters in Canada, Mexico, and the United States. Amsterdam: John Benjamins.

BüHLER, Hildegund. (1986) "Linguistic (semantic) and extra-linguistic (pragmatic) criteria for the evaluation of conference interpretation and interpreters." Multilingua 5:4, pp. 231-235. 
CHIARO, Delia \& Giuseppe Nocella. (2004) “Interpreters' perception of linguistic and non-linguistic criteria affecting quality: a survey through the World Wide Web.” Meta 49:2, pp. 278-293.

Collados Aís, Ángela. (1998) La evaluación de la calidad en interpretación simultánea: la importancia de la comunicación no verbal. Granada: Comares.

Collados Aís, Ángela; Esperanza Macarena Pradas Macías; Elisabeth Stévaux \& Olalla García Becerra (eds.) (2007) La evaluación de la calidad en interpretación simultánea. Parámetros de incidencia. Granada: Comares.

Collados Aís, Ángela; Emilia Iglesias Fernández; Esperanza Macarena Pradas Macías \& Elisabeth Stévaux (eds.) (2011) Qualitätsparameter beim Simultandolmetschen. Interdisziplinäre Perspektiven. Tübingen: Narr Verlag.

Duranti, Alessandro. (2000) Antropologia del linguaggio. Roma: Meltemi.

ERRICO, Elena \& Mara Morelli. (2012) "La percepción de la calidad en interpretación consecutiva: un estudio de caso sobre estudiantes en prácticas", comunicación presentada en el I Congreso DIDtrad, Universidad Autónoma de Barcelona, 21-22 de junio de 2012.

ERRICO, Elena \& Mara Morelli. (2013). "La calidad percibida en interpretación consecutiva: un estudio en ámbito social con estudiantes en prácticas." En: Barranco Droege, Rafael; Esperanza Macarena Pradas Macías \& Olalla García Becerra (eds.) Quality in Interpreting: Widening the Scope. Granada: Comares, vol. 2, pp. 61-81.

GARzonE, Giuliana. (2003) "Reliability of Quality Criteria Evaluation in Survey Research." En: Collados Aís, Ángela \& María Manuela Fernández (eds.) La evaluación de la calidad en interpretación: investigación. Actas del I Congreso Internacional sobre evaluación de la calidad en interpretación de conferencias. Granada: Comares, pp. 23-30.

GILE, Daniel. (1990) "L'evaluation de la qualité de l'interprétatíon par les délégués: une étude de cas." Interpreters’ Newsletter 3, pp. 66-71.

GILE, Daniel. (1995) "Fidelity Assessment in Consecutive Interpretation: an Experiment." Target 7:1, pp. 151-164.

KIRALY, Donald. (2000) A Social Constructivist Approach to Translator Education: Empowerment from Theory to Practice. Manchester: St. Jerome Publishing.

KuRZ, Ingrid. (1993) "Conference interpretation: Expectations of different user groups.” The Interpreters' Newsletter 5, pp. 13-21.

MORELLI, Mara. (2008) "La evaluación y la autoevaluación en la formación de intérpretes.” En: VV. AA. (eds.) Actas del Congreso de la Asociación Asele 2007. Alicante: Universidad de Alicante, pp. 441-448.

Morelli, Mara. (2010) La interpretación español-italiano. Planos de ambigüedad y estrategias. Granada: Comares. 
MOSER, Peter. (1995) Survey: Expectations of Users of Conference Interpretation. Final Report, commissioned by AICC. Wien: SRZ Stadt- und Regionalforschung $\mathrm{GmbH}$.

VAlero-GarCÉs, Carmen \& Anne Martin. (2008) Crossing borders in community interpreting. Definitions and dilemmas. Amsterdam \& Philadelphia: John Benjamins.

\section{Apéndice: el cuestionario}

Estamos llevando a cabo un estudio sobre la calidad de la interpretación consecutiva. Por favor, conteste a las siguientes preguntas. Gracias por su colaboración.

Elena Errico Università di Sassari - Mara Morelli Università di Genova

1) Asigne una puntuación de 1 (no adecuado) a 10 (excelente) a los siguientes parámetros relacionados con la voz de las/los intérpretes:

\begin{tabular}{|l|l|}
\hline Volumen & \\
\hline Velocidad & \\
\hline Entonación & \\
\hline Pronunciación & \\
\hline Acento & \\
\hline
\end{tabular}

2) ¿Cuál es su evaluación global acerca de la calidad de la voz de las intérpretes en una escala de 1 (muy desagradable) a 10 (muy agradable)?

3) Si tuviera que describir la actitud de las intérpretes la definiría como: (puede marcar más de una opción):

\begin{tabular}{|l|l|l|l|l|l|}
\hline Distanciamiento & & Ansiedad & & Entusiasmo & \\
\hline Interés & & Alegría & & Desánimo & \\
\hline Empatía & & Desinterés & & Nerviosismo & \\
\hline Temor & & Inseguridad & & Tristeza & \\
\hline
\end{tabular}

4) En una escala de 1 a 10, ¿cómo evalúa la actitud global de las intérpretes con respecto a la interpretación que están realizando? (1: muy negativa; 10: muy positiva). 
5) Asigne una puntuación de 1 (inadecuado) a 10 (adecuado) a los parámetros siguientes. Si no es capaz de hacer una evaluación, por favor, no rellene la casilla.

\begin{tabular}{|l|l|l|}
\hline $\begin{array}{l}\text { Control de los gestos } \\
\text { (miradas, contacto visual, postura, } \\
\text { etc.) }\end{array}$ & Terminología & \\
\hline Fluidez de exposición & Contenido & \\
\hline Cohesión lógica del discurso & $\begin{array}{l}\text { Estilo adecuado } \\
\text { (científico, coloquial, etc.) }\end{array}$ & \\
\hline Fidelidad con respecto al original & & \\
\hline
\end{tabular}

6) a) ¿Ha habido ocasiones en las cuales las voces de los participantes se superponían?

Sí $\square$ No $\square$

b) En caso afirmativo, ¿las intérpretes han intervenido para reestablecer los turnos de palabra?

Sí $\square$ No $\square$

7) a) ¿Ha habido malentendidos de tipo cultural?

Sí $\square$ No $\square$

b) En caso afirmativo, ¿las intérpretes han intervenido para solucionarlos?

Sí $\square$ No

8) En una escala de 1 a 10, puntúe la impresión de profesionalidad que le han dado las intérpretes: (1: profesionalidad nula; 10: profesionalidad elevada)

9) En una escala de 1 a 10, puntúe la impresión de fiabilidad que le han dado las intérpretes:

(1: fiabilidad nula; 10: fiabilidad elevada)

10) En una escala de 1 a 10, puntúe la calidad global de la prestación de las intérpretes:

(1: muy escasa; 10: excelente). 


\section{$\underline{\text { Sus datos }}$}

Profesión:

Nacionalidad:

Edad:

Conocimiento del español: nulo, básico, bueno, excelente, nativo

Conocimiento del inglés: nulo, básico, bueno, excelente, nativo

Conocimiento del italiano: nulo, básico, bueno, excelente, nativo

Más comentarios 


\section{NOTAS BIOGRÁFICAS / BIONOTES}

ELENA ERRICO se licencia en Interpretación de Conferencias por la Universidad de Bolonia (campus de Forlì) en 1998 y se doctora en Lenguas y Culturas Comparadas por la Universidad de Módena en 2008. Ha sido intérprete de conferencias y traductora técnica freelance y ahora es investigadora a tiempo completo de Lengua Española y Traducción en la Universidad de Sassari. Sus líneas de investigación abarcan temas de didáctica de la interpretación y lingüística del contacto entre inglés y español.

ELENA ERRICO holds an MA in Interpreting from the University of Bologna (Forlì campus) and a Ph.D. in Comparative Languages and Cultures from the University of Modena. From 1998 to 2012 she was a freelance conference interpreter and technical translator. She is currently researcher in Spanish and Translation Studies at the University of Sassari. Her research interests cover interpreter training and contact linguistics between English and Spanish.

Intérprete de conferencias, profesional libre del sector de 1991 a 2003. Desde 1991 MARA MORELLI ha sido profesora contratada e invitada en diferentes universidades e instituciones italianas y extranjeras en temas relacionados con la interpretación, la traducción y la mediación. Doctora de investigación en Traducción e Interpretación (Universidad de Granada) con una tesis sobre la resolución de la ambigüedad en la interpretación simultánea español-italiano. Actualmente es investigadora en el Departamento de Lenguas y Culturas Modernas de la Universidad de Génova. Sus líneas de investigación abarcan la interpretación, la mediación, en especial con un trabajo de campo sobre lenguas, comunicación, interpretación y mediación comunitaria.

MARA MORELLI worked as a freelance conference interpreter from 1991 until 2003. She has lectured in various subjects related to interpreting, translation and mediation in many universities in Italy and abroad. She holds a Ph.D. in Translation and Interpreting from the University of Granada, where she presented a dissertation on a study of ambiguity in simultaneous interpreting from Spanish into Italian. At the moment she is a researcher at the Department of Modern Languages and Cultures (University of Genoa). She is currently carrying out research in both domains of interpreting and mediation, in particular with a field project on language, communication, interpretation and community mediation. 